\title{
The case of sculpting atmospheres: towards design principles for expressive tangible interaction in control of ambient systems
}

Received: 13 December 2004 / Accepted: 20 September 2005/Published online: 29 November 2005

(C) Springer-Verlag London Limited 2005

\begin{abstract}
According to the vision of Ambient Intelligence, technology will seamlessly merge into people's everyday activities and environments. A challenge facing designers of such systems is to create interfaces that fit in people's everyday contexts and incorporate the values of daily life. This paper focuses on tangible expressive interaction as one possible approach towards linking everyday experiences to intuitive forms of interaction and presents a number of principles for expressive interaction design in this field. A case study of a tangible expressive interface to control a living room atmosphere projection system (orchestrating living room lighting, audio and video-art) is presented to illustrate and reflect upon the design principles. Furthermore, the case study describes possible techniques towards integrating the design principles into a design method.
\end{abstract}

Keywords Tangible expressive interaction $\cdot$ Design principles

\section{Introduction}

Two emerging trends in interaction design, common to home ambient system control, point towards new

\footnotetext{
P. Ross $(\bowtie)$

Department of Industrial Design, Eindhoven University

of Technology, Den Dolech 2, 513, 5600 Eindhoven,

The Netherlands

Tel.: + 31-40-2475966

Fax: + 31-40-2475376

E-mail: p.r.ross@tue.nl

URL:http://www.idemployee.id.tue.nl/p.r.ross/

D. V. Keyson

Faculty of Industrial Design Engineering, Delft University of

Technology, Landbergstraat 15, 2628 Delft, The Netherlands

Tel.: + 31-15-2783374

Fax: + 31-15-2787179

E-mail: d.keyson@io.tudelft.nl

URL:http://studiolab.io.tudelft.nl/keyson/
}

opportunities for the design of tangible interfaces, namely, the shift from technology use to technology presence and the transition from task-oriented to experience-driven design.

\subsection{From 'use to presence'}

The vision of Ambient Intelligence promises a seamless integration of computational technology into our everyday lives. Technology would no longer claim our attention as it does now, but rather seamlessly merge into people's everyday activities and environments [15]. Hallnäs and Redström [3] describe this change of technology's relation to people as a shift from 'use' to 'presence'. A 'use' centered view focuses on functional aspects of a system or device. Viewing a device or system from a 'presence' perspective touches upon a broader, existential definition of design in the life of a person. For example, a mobile phone can be described from a 'use' perspective by listing functional aspects (e.g., dialling, ringing, opening an audio channel, keeping an address book). From a 'presence' perspective, mobile communication technology offers a feeling of social connectivity and mobile phones are a means of personal expression [4].

\subsection{From task-oriented to experience driven design}

In considering activities in the home living context, one needs to look beyond supporting the functional aspects of the task at hand. Considering interactive systems, the total experience of fulfilling a task should be taken into account. Social, personal, and emotional engagement as well as expression are salient factors in interaction, along with ease of operation and efficiency. For ambient systems to be accepted in a home living context, values beyond system functionality need to be taken into consideration, such as playfulness, creativity and personal expression. 
In the current paper, it is argued that many previous approaches towards the design of ambient systems have incorporated one of the two above trends, but have not necessarily pursued an integral approach in which the technology presence and interaction experience are dually taken into account. Most of the ambient systems in a home context have a display-only function and do not offer or provide the user with a level of interactive control on the input side, such that creativity and personal expression are fostered. There seems to be asymmetry between the input and output modalities of interaction. For example, in the Digital Family Portraits system [8], the everyday activities of older family members are shown in expressive photoframes to provide younger relatives with a sense of peace of mind. The photoframes themselves do not offer the possibility of expressing a response to the displayed information. Ambient systems focusing on the user experience and personal creativity often focus on the interaction for the sake of the interaction experience, similar to a game or interactive art, but do not attempt to integrate this into the life of everyday users to support certain tasks. An example of such a system is the BioMuse [12] installation that picks up bioelectrical signals from muscles of the body and translates them to a sound and video-scape. The BioMuse system is intended as a performance art installation, offering both the performer and his audience an engaging experience. The system is not linked to a daily home task or activity.

This paper argues that tangible interaction can provide a means to integrate the two above mentioned trends in ambient system interfaces, focusing on interfaces that offer personal expression situated in a home environment. However, design guidelines towards creating such interfaces are lacking. This article thus has two main goals: (1) to illustrate how technology presence and experience driven design can be integrated through an expressive tangible interface design and (2) to formulate design principles towards designing such interfaces.

\subsection{Tangible interaction}

In the late 90s pioneering studies on tangible interaction concentrated on the potential of tangible interfaces to represent and manipulate virtual data [13]. For example, in Ullmer et al. [14] physical blocks were used to control and represent chunks of data. These examples represented a strong productivity-oriented view to interaction: the main goal of these interfaces was to make data handling tasks easier and more intuitive by using physical form and constraints.

More recently, Overbeeke et al. [11] described the potential of tangible interfaces towards creating a richer interaction experience, incorporating emotional expression in tangible interaction. Their view of rich interaction advocates capitalizing on a human being's natural emotional expressiveness of physical actions in a tangible interface design. The physical interaction possibilities of a product can be designed such that they offer a person freedom to express emotion, alongside functional information, in his actions. Tangible interaction becomes expressive tangible interaction.

Examples of expressive tangible interaction can be found in the alarm clock prototype developed by Wensveen [16], the musical device 'Moto' developed by [7], and the affective telephone by Fagerberg et al. [5]. A common design dimension across these examples is that expressive physical control actions are possible. The interactive alarm clock elicits actions using twelve sliders that allow the alarm time to be set in a myriad of ways. The alarm clock interprets the emotional state of the person interacting, based on parameters such as speed, order and result of the actions on the sliders. A person for example, may slowly and carelessly adjust the sliders while in a relaxed mood, but firmly and precisely set the sliders when suffering from stress.

Despite the wealth of creative ideas for designing tangible interfaces, as illustrated by the examples mentioned above, there appears to be a lack of generic design knowledge for creating an ambient system user interface that affords such rich user expression and interactivity. To facilitate the design of expressive tangible interfaces, this paper presents a number of design principles for expressive control in ambient systems. The design principles are based on experience gained from several case studies and literature. Following the design principles, an extensive case study is described, where the design principles were applied and evaluated in relation to the design of a tangible expressive interface that was used to control a living room atmosphere system. The resulting list of design principles should not be seen as exhaustive or complete, or as a complete design methodology. The principles highlight important and interesting areas of attention and insights that the designer of an expressive ambient system should consider.

\section{Design principles for an expressive tangible interface}

A number of key design principles should be considered in realizing a tangible design for ambient control that affords personal expression and takes into account technology presence in a home context. These design principles, relating to human expressiveness and ambient technology, evolved from literature and previous tangible interaction design studies and include the following:

\subsection{Consider the context of interaction}

Before designing the physical properties of an expressive tangible device for the ambient home, one should take into account the context in which the ambient control device will be used. An interface that is always present in the home environments should be considered an integral part of home life. This poses demands on the look and feel of the physical part of the interface as part of the interior. 
Furthermore, the ambient device may become a part of the daily rituals and thus should fit into daily home activities. Questions that go beyond the actual interface use need to be considered here. For example, what happens when the product is not being used actively? What is its relation to other home products and systems?

\subsection{Map and relate human expressiveness to physical interaction possibilities}

Four steps are crucial in designing expressive tangible interaction:

- Understand what a person may wish to express via the interface, given common descriptive dimensions.

- Devise a model that allows a computational system to describe the human expressiveness.

- Design physical interaction possibilities that allow a person to express the relevant expressive dimensions.

- Design a model to interpret the expressive behaviour elicited by the physical device.

In the example of the alarm clock prototype [16], a person expresses a general mood and urgency while setting the alarm time. This mood is interpreted, by the alarm clock in terms of a three-dimensional ArousalValence-Urgency model. The twelve sliders provide a person with the means to express these three dimensions in interaction. The clock's software uses a set of algorithms that map movements of the sliders to the three dimensional Arousal-Valence-Urgency model.

The case study, treated later on in this paper, will demonstrate possible techniques for applying these four steps in a new application.

2.3 Capitalize on the expressiveness of form, materials and movement

As Djajadiningrat [2] pointed out, use of form, materials and movement of physical objects can serve as a strong means to convey expression and offer a rich palette that can be used to elicit expressive actions and create meaning in interaction.

Physical objects can have a rich variety of expressive properties, such as weight, texture and elasticity. More complex constructs of objects have properties like, e.g., degrees of freedom, friction and relative position. Dynamic systems include speed, inertia, etc. A door, for example, allows different expressive ways of shutting it. A person can close it gently or slam it shut. In the latter case, the door allowed frustration to be expressed via interaction (although the door does not naturally communicate this information to an ambient system).

\subsection{Make actual experience pivotal in the design process}

The complex nature of experiential factors that may influence interaction with an ambient system may be difficult for a designer to perceive. Real-life 'experiential' testing is crucial to learn how design decisions may influence the interaction experience of people. This does not mean that every mock-up in the design process needs to be fully functional. A 'Wizard of Oz' approach, with which system functionality is simulated by a human controller, can result in interaction experiences as if the mock-up was fully functional. Furthermore, the testing needs to take place in a context that is as similar as possible to the intended context of use. It is almost impossible to predict how a product will elicit expressive behaviour if it only exists on paper or on a computer screen. Physical models and tests in context are simply essential for designing expressive tangible interaction.

\subsection{Consider the subjective nature of expression}

Expressive interaction is essentially subjective. In ambient systems, subjectivity is salient in two ways: an individual's experience of the ambient system's output is subjective and the expressive input of individuals needs to be interpreted by the system, taking individual differences into account.

When designing an ambient system, it is important to realize that to a certain degree, each individual may interpret the expressiveness of a system or device differently. Secondly, the physical actions of a person have an individual nature, like handwriting or sculpting clay, since every individual has a different perceptual-motor system. Even within a particular person differences in expression may occur over time due to factors such as refinement of motor skills, mood changes and motivation. Thus it is important that the expressive interface allows for individual creativity and fluctuations in expression. For example, the alarm clock sliders offer freedom of interaction. Subjects in its user study expressed Valence, Arousal and Urgency slightly differently, but were found to be generally consistent in their individual behaviour [16]. Similarly, in work by Hummels et al. [7] freedom of interaction was considered a key feature in allowing people to express themselves. Finally, the system software model needs to be designed in such a way that individual fluctuations and personal preferences are taken into account when interpreting the expressive behaviour. Ideally, the system should have a learning potential, to gradually grow towards knowing an individual's personal style and developments in personal expression.

\section{Case study}

This section describes a case study that was conducted to consider how each of the above design principles could be applied to the design of an expressive tangible interface. The case study pursued two design goals. One goal was to create intuitive and expressive control over complex functionality of a home ambient system. The 
other goal was to offer a style of interaction that could fit in a home context, with support for personal expression. The design principles were also used to structure the analysis of results obtained from a series of user studies leading up to and involving the end design.

\subsection{An expressive tangible living room atmosphere controller}

An expressive tangible interface was considered suitable for controlling a living room atmosphere projection system, managing lights, sound and video-art projected on the wall. A rich interactive tangible interface was desired here considering the potential it has in expressing an affective phenomenon like atmospheres and the possibility to design it such that it would fit in to the home environment.

Figure 1 shows the system architecture. The atmosphere projection system was built in the Studio Home lab at Delft University of Technology, to create a living room like feel. This case study was conducted as part of the Residential Gateway Environment project [1].

\subsection{Design steps}

Once the home lab atmosphere projection hardware was set up, the following steps were taken, leading to the design of the expressive interface for atmosphere control:

1. Atmosphere scaling: Studies were conducted involving collages and semantic scaling to determine underlying dimensions in experience of atmospheres.

2. Functional atmosphere projection system: The hardware set up was built and a multi-dimensional navigational space was created based on semantic scales from step 1. This space was filled with sample atmospheres to create a functional atmosphere projection system.

3. Gesturing workshop: Designers were asked to make gestures corresponding to display changes along the atmosphere dimensions in a study to define the initial physical parameters of the tangible interface.

4. Development of interface through mock-ups and user evaluation: Physical modelling workshops were conducted with designers. Design decisions were evaluated through testing the mock-ups in context.
5. Design and realization of a fully functional prototype of the expressive tangible interface.

6. User studies involving the functional prototype. Below is a brief description of the design work conducted under each of the above six steps.

\subsubsection{Step 1: atmosphere scaling}

As outlined in design principle number 2 ("Map and relate human expressiveness to physical interaction possibilities"), the expressive dimensions of the ambient system were sought through a series of empirical studies. Subjects judged mood boards of atmospheres along 13 dimensions in a semantic differential experiment [10]. Results of a factor analysis yielded three primary factors, labelled Activity level, Warmth level and Attention level.

\subsubsection{Step 2: create a functional atmosphere projection system}

The three resulting dimensions were used to create a three-dimensional scaling model describing experience of atmospheres as depicted in Fig. 2. The model was subsequently used to arrange video-art, light and music content along the three atmospheres scaling dimensions, resulting in coherent atmospheres (Fig. 3). The video art was designed in cooperation with a professional videojockey, who had experience in creating atmospheres by use of video material. The color schemes were loosely based on work on color and associations with moods [9]. The music was selected using rules of thumb concerning, e.g., tempo and genre.

Considering the home context of interaction (principle 1), a number of design criteria emerged.

- The tangible expressive device should be a decorative element in the interior and become an integral part of it, even when it is not actively being used.

- The device should fit in people's rituals to create atmospheres, not replace it. A study conducted by Kuiper-Hoyng and Beusmans [6] gave insight into rituals that people have in creating atmospheres (e.g., cleaning up, lighting candles and burning incense). It appears appropriate to have the device enrich these rituals rather than replace them.
Fig. 1 Scheme of the atmosphere projection system architecture
Studio Home Lab

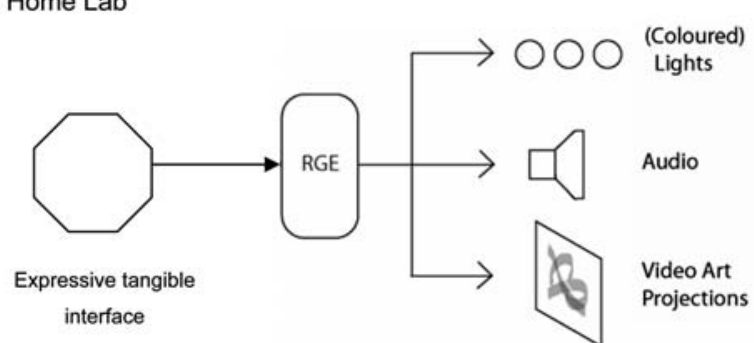




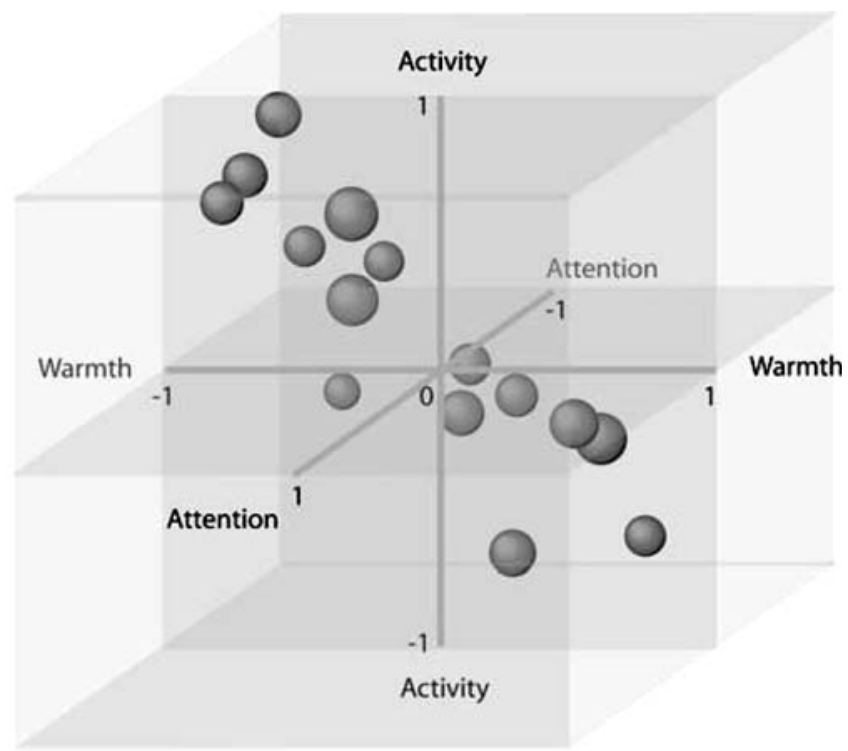

Fig. 2 Depiction of atmosphere space with the three scaling dimensions activity, warmth and attention. The spheres represent collections of audio, lighting and video art content placed as atmospheres in the model

- The device should enable multiple people to set the atmosphere, considering a house often has multiple residents with different personal tastes.

\subsubsection{Step 3: gesturing workshop}

Gestures were used to find the basic physical action possibilities of the tangible interface. Designers, situated in the home lab with the atmosphere projection system, were asked to express the dimensions of atmospheres (warmth, activity and attention) across a range of points along the atmosphere model (Fig. 4).

The observed gestures across designers were then categorized under each of the three model dimensions as shown in Table 1.

\subsubsection{Step 4: development of interface through mock-ups and user evaluation}

A modelling workshop was held immediately after the gesturing workshop, with the same participants. The results of this exercise led to several early mock-ups that were used to further guide the design process. Approximately 20 basic mock-ups were constructed, during an iterative trial and error process. The simple cardboard mock-ups were evaluated in context using the Wizard of $\mathrm{Oz}$ approach, in combination with the atmosphere projection system, as described in design principle 4 .

A final mock-up with basic hardware functionality and forms that could be expressively manipulated (Fig. 5) was created and evaluated.

In the translation process from gestures to a tangible expressive product, the expressive potential of material, form and movement was pivotal (principle 3). The resulting mock-up could be described as resembling an abstract sculpture that allows people to dynamically sculpt a home atmosphere. The main elements were a rotating disc and four flags. Each flag had two degrees of freedom, i.e., the flag pitch and a rotation around its axis. Material expression was used on the flags such that one side was covered with soft patching and one side with aluminum. A geared DC motor with controllable rotation speed, drove the stage. By changing the flag positions and setting the rotation speed expressive patterns could be created. Furthermore, the flags and the stage allowed expressive actions ranging from subtle and smooth to fast and coarse. Table 2 shows a number of design considerations regarding how the results of the gestures session were translated into the mock-up's interaction possibilities.

Pilot testing of final mock-up As stated in principle 4, actual experience of interaction should guide the design process. At this point, the general concept of the interface was conceived. The concept was highly innovative, which made it difficult to assess whether it would work or not. Therefore, a small explorative study was conducted to see to what degree the final mock-up design could enable people to express a range of atmospheres and to see what types of expressive actions would be conducted in relation to changes in the atmosphere display system along the three model dimensions of warmth, activity and attention.

Five subjects' actions were analysed visually in relation to changes in the atmosphere. Some variation between subjects was found, but several distinct trends emerged in subjects' behaviour across changes in dis-
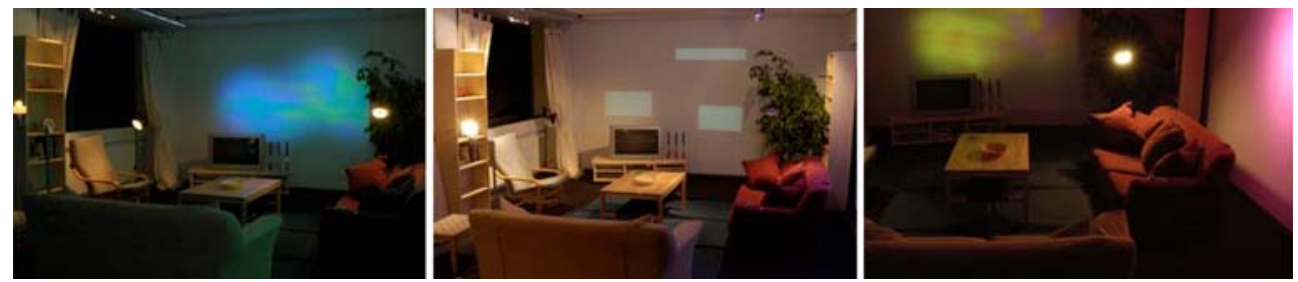

Fig. 3 Home living room lab with several projected atmospheres. Left picture a calm atmosphere (medium Warmth, low activity, medium attention), created by a subtly animated blue figure on the wall; Middle picture an active atmosphere, created by moving blocks on the wall and bright lights; Right a warm atmosphere, created by reddish lights, video art of similar colours and use of spotlights 
Fig. 4 Examples of expressive gestures made during the gesturing workshop, relating to the atmosphere projections along the model dimensions of activity, warmth and attention
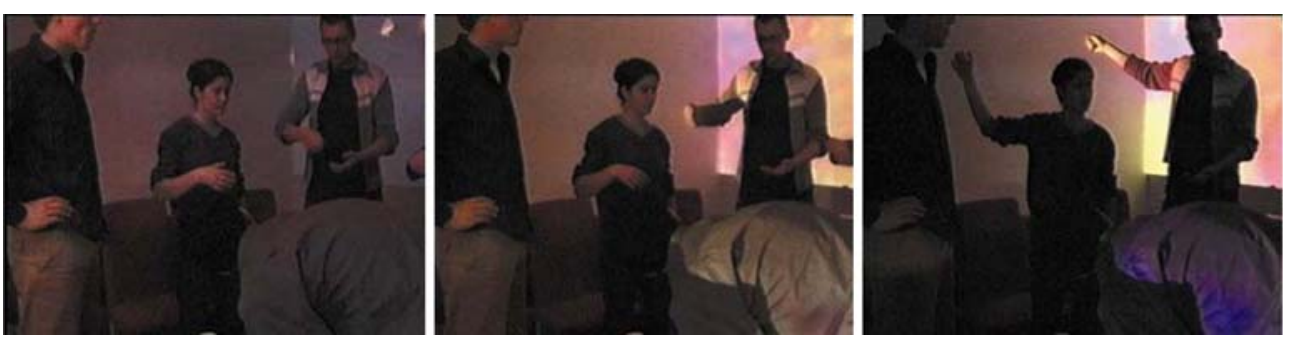

Table 1 Summary of gestures made to express the activity, warmth and attention level across a range of projected atmospheres in the model space

\begin{tabular}{|c|c|c|}
\hline Activity & Warmth & Attention \\
\hline Finger drumming rate & Proximity of gestures to body & Number of actions \\
\hline Engagement of more limbs & Nurturing or cupping-like gestures & Gestures in focal or peripheral area of vision \\
\hline Amount of squeezing tension & $\begin{array}{l}\text { Duration of contact time } \\
\text { (longer means higher warmth) }\end{array}$ & Fine versus course movements \\
\hline Hand or arm rotation speed & Inner versus outer side of hand contact area & $\begin{array}{l}\text { Height differences in gestures } \\
\text { (more height to convey more attention) }\end{array}$ \\
\hline Speed of gestures & Use of breath & \\
\hline
\end{tabular}

played atmospheres. Participants generally expressed: (a) higher activity levels via higher stage rotation speeds, (b) warmer atmospheres by turning the soft side of the flags up and (c) more exuberant atmospheres (i.e., high activity and high attention levels) by creating upright patterns. Variation was found mainly when inspecting the exact configurations of individual flags.

\subsubsection{Step 5: Design and build a fully functional proto- type of the expressive tangible interface}

A fully functional prototype based on the final mock-up was created, equipped with sensors and read-out software, to enable more detailed user testing. The main elements, the rotating stage and the four flags, remained. The overall design, look and feel of the prototype were improved, making the prototype a finished product that had decorative value for the living room. The key features were the following:

- Rotation speed of the platform could be adjusted by grasping the platform.

- The flags had a palm wood surface on one side, and an aluminum surface on the other, to create warm and cold sides.

- A slot was created in the stage platform to enable future insertion of an RF ID tag, so that the user's individual settings of the tangible interface in relation to specific atmospheres could be recorded (Fig. 6).
Fig. 5 The final tangible interface mock-up with multiple degrees of movement freedom to express a spectrum of atmospheres
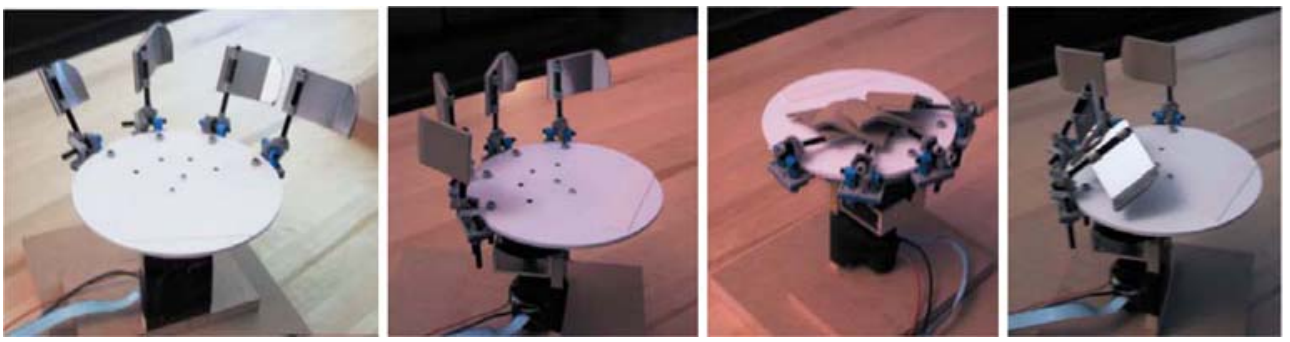

Table 2 Examples of the gestures session's results and their translation into mock-up interaction possibilities

\begin{tabular}{|c|c|c|}
\hline Activity & Warmth & Attention \\
\hline Gesture: Hand or arm rotation speed & Gesture: nurturing or cupping-like gestures & Gestures: number of actions \\
\hline Mock-up: variable speed of rotating stage & $\begin{array}{l}\text { Mock-up: flags can form closed } \\
\text { and smooth cupping-like patterns }\end{array}$ & $\begin{array}{l}\text { Mock-up: freedom to create patterns } \\
\text { in one go or in any number of actions }\end{array}$ \\
\hline Gesture: Speed of gestures & Gesture: inner versus outer side & Gestures: fine versus coarse movements \\
\hline $\begin{array}{l}\text { Mock-up: flags that can be moved } \\
\text { with variable speeds }\end{array}$ & $\begin{array}{l}\text { of hand contact area } \\
\text { Mock-up: a soft (warm) side } \\
\text { and a metal (cold) side on the flags. }\end{array}$ & $\begin{array}{l}\text { Mock-up: freedom to manipulate the flags } \\
\text { with fine or coarse actions }\end{array}$ \\
\hline
\end{tabular}


Fig. 6 Top left Final prototype of expressive tangible interface prototype, named 'Carrousel'. An insertion point in stage platform for detecting optically encoded IDtokens is visible. Right column two different expressions made with the Carrousel. Bottom left the inside of prototype with DC motor, drive belt and battery powered infrared unit for transmitting flag settings to the base print on the chassis
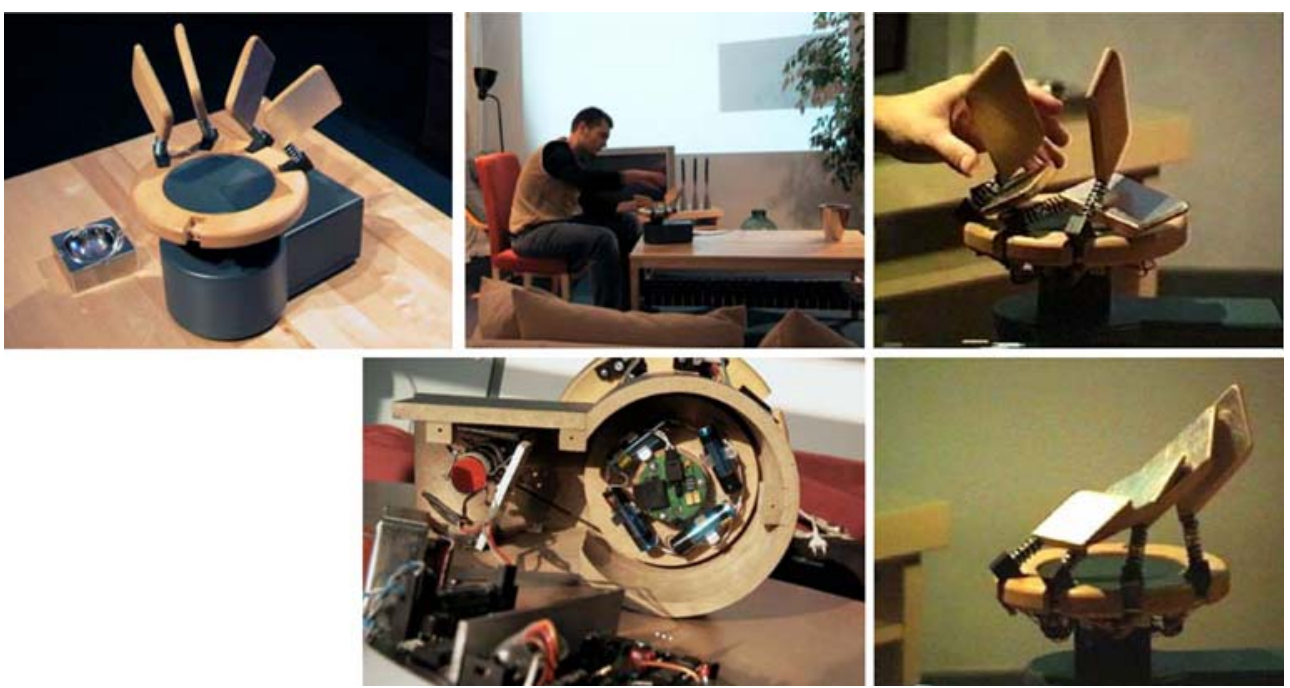

- The prototype was engineered mechanically and electronically: each flag was equipped with an encoder and a potentiometer to sample its position. The DC motor was enclosed in a non-obtrusive housing and all electrical wiring was hidden from view. The readout of the sensors in the flags was sent via battery powered infrared wireless communication to enable communication of the flag settings to the base unit. The prototype transmitted the current flag orientations and rotation speed to a central computer through the serial port at a frequency of $25 \mathrm{~Hz}$. An on/off switch and serial connector was placed at the back of the base unit.

- The prototype was dubbed 'Carrousel'.The Carrousel transmits raw data to a central computer, including flag pitch and roll and stage rotation speed, as depicted in Fig. 7.

These raw variables, however, gave no indication of the expressive qualities of actions and their resulting flag patterns. It was necessary to enable the system to see the expression in the behaviour and the resulting patterns like a human being would. Parameters describing these expressive qualities were derived from a user study by visual inspection of behaviour on the prototype and subsequently approximated using geometric calculation. The expression parameters are listed in Table 3.

\subsubsection{Step 6: conduct user studies involving the functional prototype}

To consider how users would map a range of projected atmospheres to the expressive dimensions of the Carrousel, two experiments were conducted using a group of seven adults who were selected for having an expressive hobby, like playing an instrument. None of the participants had a background in design. In the first experiment, seven basic atmospheres were displayed with fourteen subtle variants of each basic atmosphere, for a total of 21 trials. Each condition was presented at random. Subjects were instructed to rate each projected atmosphere along 13 semantic scales, describing expression of atmospheres. Analysis of these ratings indicated the level of activity, warmth and attention that a subject attributed to a certain atmosphere. These data
Fig. 7 Flag Pitch and Roll, as one of the measurements of user input, was summarized via two variables, the Pitch $\left(0^{\circ}-110^{\circ}\right)$ of the axis of the Flag $(\mathrm{Pn})$ and the Flag rotation $\left(0^{\circ}-360^{\circ}\right)$ or Roll along its axis $(\mathrm{Rn})$
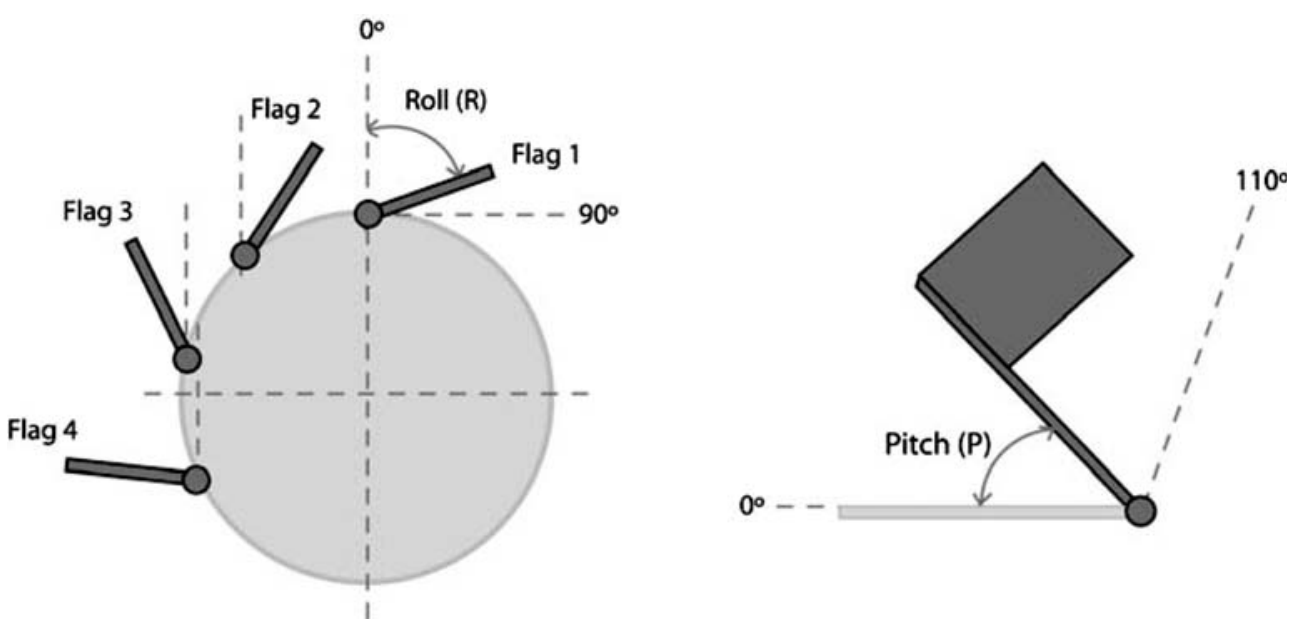
Table 3 parameters of expression used to interpret expressive behaviour on the Carrousel

Pattern parameters

Openness

Uprightness

Smoothness

Which Side Up

Rotation Speed

Action Parameters

Sample Time

Action No
The mean distance between Flag tips. Range $[0,1]$

The mean pitch of the Flags. Range [0,1]

Mean difference in orientation between a Flag and its adjacent Flag. Range $[0,1]$

Dominant Flag surface in view. Range [0,1]. $0=$ all metal sides up, $0.5=$ neutral, $1=$ all wooden sides up

Stage rotation speed. Actual speeds range from 3 to $30 \mathrm{rpm}$. Parameter range $=[0,1]$

The time from the first action to the last in setting an atmosphere, measured in seconds

Number of Carrousel handling actions for expressing one atmosphere preset, including actions

for controlling rotation speed of the stage. were used to relate the expressive behaviour on the interface in a second experiment to the atmosphere dimensions.

In this second experiment, conducted one week later, subjects were asked to use the tangible interface to express the same atmospheres that were presented to them in the first experiment. Duplications of the seven basic atmospheres were added as a reliability test, resulting in a total of 28 presented atmospheres. The flag orientations and the rotation speed were sampled and logged at 25 times per second. Based on the collected data, the parameters were calculated, as described in Table 3 . The results from these calculations were mapped to the participants' subjective ratings for warmth, attention and activity across all atmospheres. In this manner, the differences between subjects in experience of atmospheres could be cancelled out and expressive behavior of all participants could be compared in relation to warmth, activity and attention values. At the conclusion of the formal trials, each participant was interviewed to obtain subjective feedback on their experience of interacting with the Carrousel and suggestions for improvements.

After these two detailed experiments, a third informal experiment was conducted at the Ubicomp2004 conference in Nottingham, England. Thirteen conference attendees and student volunteers participated in this informal study. The Carrousel actually controlled the atmospheres in this case and used mapping algorithms based on the sculpting preferences of one of the authors. The participants were asked to create certain atmospheres and their behavior was observed.

\subsection{Results and discussion of experiments}

In this section, the results of all three experiments are combined and discussed. The results treated here originate mostly from the first two studies. When results of the informal study are treated, this is explicitly mentioned.

Expression of atmospheres The Carrousel appeared to enable the participants to express the presented range of atmospheres. The subjects were able to translate their experience of the presented atmospheres into form, material and movement expression on the Carrousel. In other words, they were able to sculpt atmospheres. The participants were consistent, since they made similar expressions on the Carrousel for similar atmospheres. Furthermore, there were significant trends in their behaviour related to warmth, activity and attention. For instance, a higher activity factor in atmospheres generally resulted in a higher rotation speed of the stage (Fig. 8).

The rotation speed turned out to be the strongest parameter to express an atmosphere dimension (activity). All participants expressed higher activity with higher rotation speed. Also, in the third experiment, all participants connected rotation speed to activity. But participants had their own way of expressing other elements of atmospheres. These differences between participants became apparent looking at the other parameters (e.g., smoothness and uprightness) in the second experiment. These parameters are also harder to relate to an atmosphere dimension than rotation speed: it is difficult to tell what kind of atmosphere a participant tried to express by analyzing these parameters. However, there are general trends visible. For

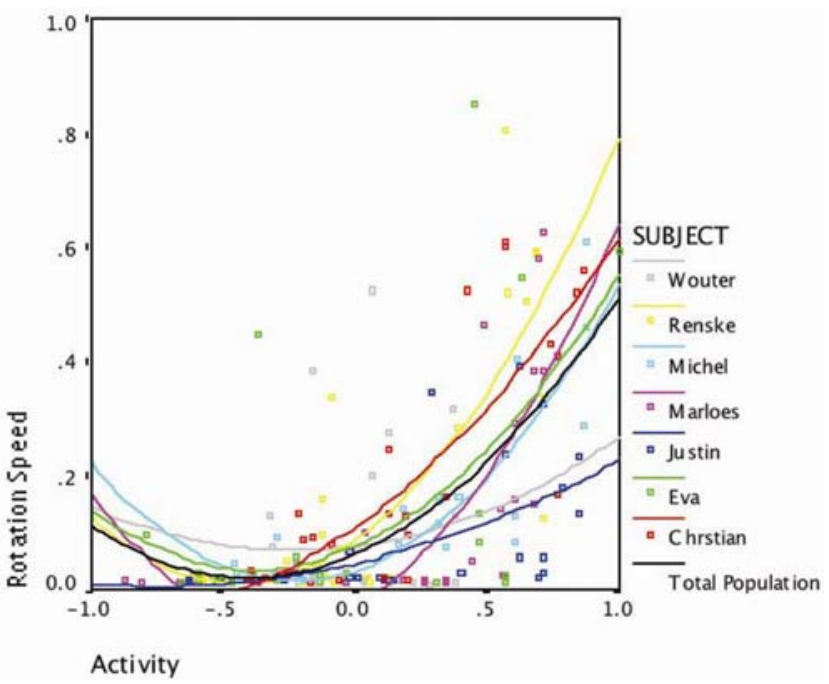

Fig. 8 Rotation speed of the Carrousel stage plotted for all participants of the second experiment against the perceived Activity of the presented atmospheres 
example, from visual inspection of the expressions of the subject Michel plotted against activity and warmth in Fig. 9, it turns out that patterns become more closed, less upright and smoother when activity and warmth increase.

Subjective nature of expressions As expected from the subjective nature of expression, differences between subjects were found both in their experience of atmospheres in terms of warmth, activity and attention, and the way they expressed these atmospheres on the Carrousel.

However, one of the subjects had a very individual style of expression to communicate desired atmospheres, which did not conform to the anticipated user behavior. This subject focused on details in the music in his expressions, rather than the general feel of the atmosphere. For example, he put the flags alternating in high and low position to express a reggae beat in the music that was part of the atmosphere. General trends in this behavior were largely absent, which made it difficult to map his behavior on the Carrousel to warmth, activity and attention values.

Interaction experience Interviews with the participants indicated that they generally were positive about the concept of this expressive tangible device in control of atmospheres. They liked the physical nature of the interaction and the feel of the materials. Interacting with the Carrousel triggered their imagination and allowed them to be creative. Most participants reported that they developed skills and discovered new 'sculpting' options as the experiment progressed. Furthermore, the participants agreed that the Carrousel blended well with the living room environment and they found it aesthetically pleasing.

Exploration and learning effects In the informal study, a single mapping model was used for all participants. Since earlier experiments pointed out that every individual sculpted in a slightly different way, the Carrousel sometimes reacted counter-intuitively for participants in this study.

In these cases, the participants were still able to find an intended atmosphere through exploration of different form, material and movement expressions on the Carrousel. After this, a learning effect emerged, which allowed them to find certain atmospheres quickly. Ideally, the Carrousel would learn from a person, instead of the other way around, but where the mapping algorithms fall short, an intended atmosphere can still be found by exploration.

Implementation of a learning, fuzzy-logic-based system for interpreting individuals' expressive behaviour related to the atmosphere dimensions would be a logical next step in the development of the Carrousel.
Fig. 9 The expressions subject Michel made plotted against the two atmosphere dimensions activity and warmth

\section{Michel}

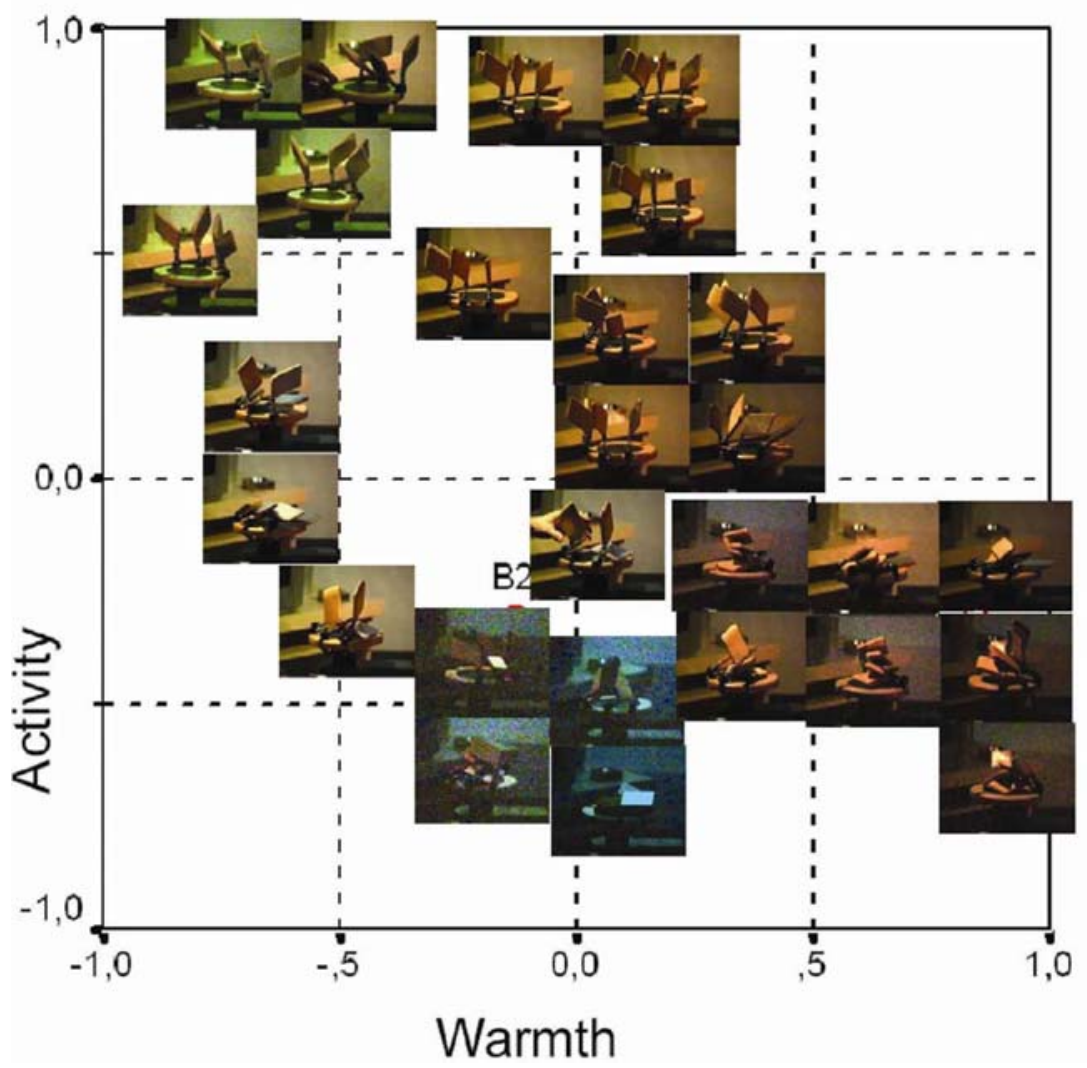




\section{Conclusions and reflection on design principles}

This paper concludes with a reflection on the role of the design principles in the presented case study, followed by a discussion on how these principles may assist future design projects and how they could be further developed.

\subsection{Role of design principles in the case study}

As mentioned in the introduction, examples of tangible expressive interfaces in home contexts are scarce and design methodologies are lacking. In the presented case study, the design principles provided guidance, which led to the design of a subjectively perceived innovative interface that produced promising results in evaluative tests: the Carrousel was shown to offer control over a complex home ambient system and was considered by subjects to provide an interaction experience that could fit in a home context.

The design principles contributed to both the design decisions and conception of the design process. For example, considering principle 5 (individual nature of expression), an ID-token slot was included in the design to allow personal identification to the system. Application of principle 1 (context) influenced material use and design to fit in with the living room environment. The user test indicated that these design decisions were useful. Regarding the design process, the design principles pointed towards the necessary elements (e.g., atmosphere modelling, mock-up designs and iterative user evaluation of mock-ups). In pointing out the necessary elements, the principles did not limit possible techniques for realizing these elements (e.g., the semantic differential experiment could be replaced by another technique if this would be appropriate for another design brief).

\subsection{Reflection on design principles}

The design principles mainly focus on creating a context for an interaction experience, rather than focusing on technological issues or ergonomic issues such as ease of use and efficiency. One could consider thus the principles to be of a qualitative and somewhat open nature. Applying these principles still requires a designer to translate this openness into a concrete design. The role of the designer also incorporates selection of relevant design techniques for applying the principles. A next step in developing the design principles would be to include suggestions for possible techniques related to the nature of the design brief.

Issues like ergonomics and technology naturally remain essential to the design of tangible expressive interfaces. It should be clear that although the principles were formulated from the conceptual point of view of expressive design, practical issues such as cost efficiency, marketing, and sustainability should also be taken into account when designing for industry.
The design principles presented in this paper are still far from constituting a full design methodology. They can, however, provide a useful basis for structuring design knowledge, generated through design practice. This design knowledge can support future design projects aimed at creating expressive control over complex ambient systems, while offering a style of interaction attuned to a given context. More often than not, it seems that designers involved with expressive, tangible interfaces for ambient control re-invent the wheel over and over again. By iteratively applying the design principles in new projects and feeding back the acquired design knowledge into the principles, a structural build-up of knowledge can be achieved in this new area of interface design.

Acknowledgements Technical infrastructure support for the case study was provided by the Residential Gateway Environment project, funded by Senter, Dutch Ministry of Economic Affairs. Philip Ross conducted the case study as his master's thesis at Delft University of Technology. The authors would like to thank Caroline Hummels, Kees Overbeeke and Aadjan van der Helm for their input in the case study, Julie Beusmans and Liliane Hoyng for work on the moodboards, V.J. Jeroen Vester for his help in creating the video art and Rob Luxen and Kerem Odabasi for excellent technical assistance. Photos of Fig. 3 by P. Musters.

\section{References}

1. Den Hartog FT, Keyson DV, Snijders F, Koonen AMJ (2004) Tackling the complexity of residential gateways in an unbundling value chain. In: Proceedings of ISSLS March 2004, Edinburg, Scotland

2. Djajadiningrat JP, Wensveen SAG, Frens JW, Overbeeke CJ (2004) Tangible products: redressing the balance between appearance and action. Special issue on tangible interaction of the J Personal Ubiquit Comput 8:294-309

3. Hallnäs L, Redström J (2002) From use to presence: on the expressions and aesthetics of everyday computational things. ACM Trans Comput Hum Interact (TOCHI) 9(2):106-124

4. Ling R (2004) The mobile connection: the cell phone's impact on society. Morgan Kaufmann, San Francisco

5. Fagerberg P, Ståhl A, Höök K (2003) Designing gestures for affective input: an analysis of shape, effort and valence. In: Proceedings of MUM 2003. Norrköping, Sweden

6. Kuiper-Hoyng LLML, Beusmans JWF (2004) Using home networks to create atmospheres in the home: technology push or (latent) user need? In: Dutch directions in HCI, Amsterdam

7. Hummels CCM, Ross PR, Overbeeke CJ (2003) In search of resonant human computer interaction: building and testing aesthetic installations. In: Rauterberg M, Menozzi M, Wesson J (eds) Proceedings of interact 2003. IOS, Amsterdam, pp 399-406

8. Mynatt ED, Rowan J, Craighill S, Jacobs A (2001) Digital family portraits: supporting peace of mind for extended family members. In: Proceedings of the SIGCHI conference on $\mathrm{Hu}-$ man factors in computing systems. ACM, New York, pp 333340

9. Ohmi G, Lee SH, Lee SH, Price BG (1996) Cross cultural study of Impressions on colors and Forms. In: proceedings of 1st Asian Design Conference. Beijing 1996, pp 323-330

10. Osgood CE, Suci GJ, Tannebaum PH (1957) The measurement of meaning. University of Illinois Press, Urbana

11. Overbeeke CJ, Djajadiningrat JP, Hummels CCM, Wensveen SAG (2002) Beauty in usability: forget about ease of use! In: Green WS, Jordan PW (eds) Pleasure with products: Beyond usability. Taylor \& Francis, London, pp 9-18 
12. Tanaka A (2000) Musical performance practice on sensorbased instruments. In: Wanderley MM, Battie M (eds) Trends in gestural control of music. IRCAM, Paris, France

13. UIImer B, Ishii H (2000) Emerging frameworks for tangible user interfaces. IBM Syst J 39(3 and 4):915-931

14. Ullmer B, Ishii H, Glas D (1998) Media blocks: physical containers, transports, and controls for online media. In: Proceedings of SIGGRAPH 98. 19-24, July (1998), ACM Press, New York, pp 379-386
15. Weiser M (1991) The computer for the 21st century. Sci Am 265(3):94-104

16. Wensveen SAG, Overbeeke CJ, Djajadiningrat JP (2002) Push me, shove me and I know how you feel. Recognising mood from emotionally rich interaction. In: Proceedings of the DIS2002. ACM Press, New York, pp 335-340 